\title{
Solid phase epitaxy in uniaxially stressed (001) Si
}

\author{
N. G. Rudawski ${ }^{\text {a) }}$ and K. S. Jones \\ Department of Materials Science and Engineering, University of Florida, Gainesville, \\ Florida 32611-6400, USA \\ R. Gwilliam \\ Nodus Accelerator Laboratory, University of Surrey, Guildford, Surrey GU2 7XH, United Kingdom
}

(Received 24 July 2007; accepted 2 October 2007; published online 22 October 2007)

\begin{abstract}
The effect of [110] uniaxial stresses up to $1.5 \mathrm{GPa}$ on defect nucleation during solid phase epitaxy of amorphous (001) Si created via ion implantation was examined. The solid phase epitaxial regrowth velocity was slowed in compression. However, in tension, the velocity was unaffected. Both compression and tension resulted in an increase in regrowth defects compared to the stress-free case. The defects in compression appear to arise from roughening of the crystallizing interface whereas in tension it is proposed that reorientation of crystallites near the initial amorphous/ crystalline interface is responsible for defect formation. (C) 2007 American Institute of Physics.

[DOI: $10.1063 / 1.2801518$ ]
\end{abstract}

Solid phase epitaxy (SPE) in amorphous $(\alpha)$ Si created via ion implantation produces enhanced dopant activation and shallower junctions. ${ }^{1}$ However, stresses found during typical device fabrication may influence the SPE process. ${ }^{2,3}$ Early investigations revealed exponential enhancement of the [001] SPE using hydrostatic pressure while subsequent investigations revealed uniaxial stress in the plane of the regrowing $\alpha /$ crystalline interface caused smaller changes to SPE rates. ${ }^{4-6}$ In-plane compression also caused significant roughening of the regrowing $\alpha$ /crystalline interface. ${ }^{7}$ This is significant since interfacial roughening is known to cause SPE-related defect formation. ${ }^{8,9}$ However, while BarvosaCarter et $a .^{7}{ }^{7}$ quantified and modeled the roughness of the regrowing interface, they did not quantify the resulting defects or examine tensile stresses. Furthermore, the stresses used were fairly small in magnitude $(<0.5 \mathrm{GPa})$ and the stresses present in $\mathrm{Si}$-based technology can be $\sim 1 \mathrm{GPa}$ or more. ${ }^{2}$ Thus, the goal of this study is to examine the effect of very high uniaxial [110] stresses on SPE in (001) Si.

For this study, 50- $\mu \mathrm{m}$-thick polished (001) Si wafers were used. The specimens were first $\mathrm{Si}^{+}$implanted at 50 and $200 \mathrm{keV}$ with doses of $1.0 \times 10^{15} \mathrm{~cm}^{-2}$ and subsequently $\mathrm{As}^{+}$ implanted at $300 \mathrm{keV}$ to a dose of $1.8 \times 10^{15} \mathrm{~cm}^{-2}$. Samples were cleaved along $\langle 110\rangle$ directions into $\sim 0.3 \times 1.8 \mathrm{~cm}^{2}$ strips. Stress was applied by bending and inserting the strips into slots in a quartz tray spaced $\sim 1.5 \mathrm{~cm}$ apart. A Philtec laser displacement measurement system accurate to $1 \mu \mathrm{m}$ measured the local radius of curvature along the strips. The bent strips were symmetric and parabolic in shape with the smallest radius of curvature at midlength. The [110] stress $\left(\sigma_{[110]}\right)$ was calculated using the [110] Young's modulus of $\mathrm{Si}$ near $\sim 500{ }^{\circ} \mathrm{C}\left(\sim 1.61 \times 10^{11} \mathrm{~Pa}\right)$, the wafer halfthickness, and the local radius of curvature as presented elsewhere. ${ }^{10,11}$ Maximum repeatable stresses of $1.5 \pm 0.1 \mathrm{GPa}$ were attained. Specimens were annealed at $525^{\circ} \mathrm{C}$ in $\mathrm{N}_{2}$ ambient for 0.7-3.2 h. Stress-free, tensile, and compressive specimens were annealed simultaneously for each anneal time. Upon removal from the quartz tray after annealing, the

\footnotetext{
a) Author to whom correspondence should be addressed. Electronic mail:
} ngr@ufl.edu specimens exhibited no detectable radii of curvature $(\gg 2.0 \mathrm{~m})$ indicating no measurable plastic strain. Regrowth of the $\alpha$-Si layers was examined using weak-beam dark-field (WBDF) cross-sectional transmission electron microscopy (XTEM). Specimens were prepared via focused ion beam (FIB) milling within a distance of $\pm 3 \mathrm{~mm}$ from the specimen centers to minimize the presence of any thermal gradient.

Figure 1(a) presents a WBDF-XTEM image of an asimplanted specimen indicating an $\alpha$-Si layer $\sim 340 \mathrm{~nm}$ deep with an initial $\alpha /$ crystalline interface with many small crystallites residing just inside the $\alpha$-Si region as observed using high-resolution XTEM (not presented). Following annealing for $1.3 \mathrm{~h}$ stress-free, shown in Fig. 1(e), the specimens exhibit a smooth SPE front with estimated root-mean-squared roughness $\left(R_{\mathrm{rms}}\right)$ of $3 \pm 2 \mathrm{~nm}$ after $250 \pm 5 \mathrm{~nm}$ of regrowth with a few SPE-related defects (indicated by arrow). The defects tend to form near the original $\alpha$ /crystalline interface and propagate upward resulting from the misoriented crystallites after amorphization. ${ }^{12}$ A small band of end of range defects is observed near the initial $\alpha /$ crystalline interface. $^{8}$ Figures 1(b)-1(d) present images of specimens annealed for $1.3 \mathrm{~h}$ with $\sigma_{[110]}=-0.5,-1.0$, and $-1.5 \mathrm{GPa}$, respectively.

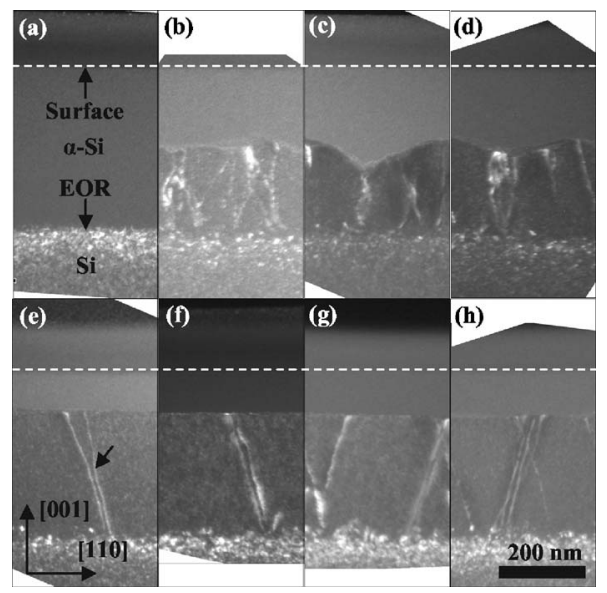

FIG. 1. (a) WBDF-XTEM image of an as-implanted specimen. WBDFXTEM images of specimens annealed for $1.3 \mathrm{~h}$ with $\sigma_{[110]}=(\mathrm{b})-0.5$, (c) -1.0 , (d) -1.5 , (e) 0 , (f) 0.5 , (g) 1.0, and (h) $1.5 \mathrm{GPa}$. 


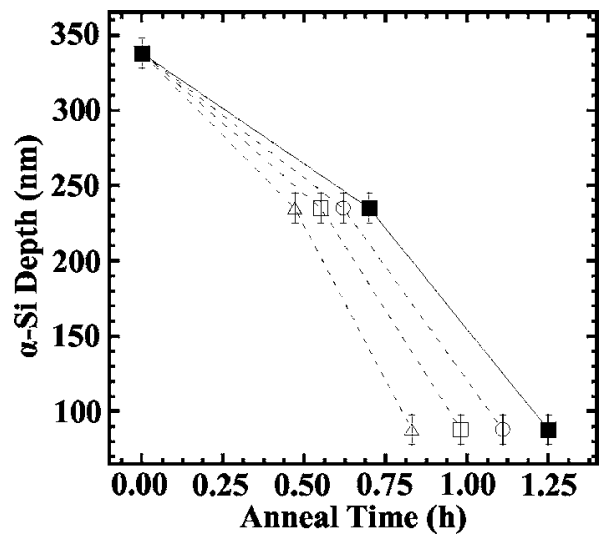

FIG. 2. Plot of measured (ם) $\alpha$-Si depth vs annealing time for four different samples with $0 \leqslant \sigma_{[110]} \leqslant 1.5 \mathrm{GPa}$ and predicted (Ref. 6) $\alpha$-Si depth vs annealing time for $\sigma_{[110]}=0.5(\bigcirc), 1.0(\square)$, and $1.5 \mathrm{GPa}(\triangle)$.

The specimens annealed with compression exhibit a resulting $\alpha /$ crystalline interface with average $R_{\mathrm{rms}}=26 \pm 5 \mathrm{~nm}$ and a large number of SPE-related defects. It appears that the defects nucleate from the initial $\alpha$ /crystalline interface, as well as within the regrown $\mathrm{Si}$. The SPE rate was retarded compared to the stress-free case, as $\sim 200 \mathrm{~nm}$ of SPE occurred which agrees with prior results. ${ }^{5,6}$ However, due to the rough interface, the amount of regrowth is approximate making quantification of the SPE rate difficult. The generation of off-axis SPE fronts during roughening which was regrown slower than the [001] front may also be a factor in this observed retardation. ${ }^{13}$ Regarding the cases of $\sigma_{[110]}=0.5,1.0$, and 1.5 GPa, shown in Figs. 1(f) and 1(g), respectively, the resulting $\alpha /$ crystalline interfaces are smooth and strikingly similar to the stress-free case in Fig. 1(e) with $R_{\text {rms }}$ $=3 \pm 2 \mathrm{~nm}$. However, more SPE-related defects nucleate from the original $\alpha /$ crystalline interface in tension compared to the stress-free case. The amounts of regrowth were very similar to the stress-free case with $250 \pm 5 \mathrm{~nm}$ of regrowth which disagrees with prior reports observing enhancement to SPE. ${ }^{5,6}$

To further examine the effect of tension on SPE rate, specimens were annealed for $0.7 \mathrm{~h}$ with $\sigma_{[110]} \geqslant 0$. In all cases, the resulting amount of regrowth was very similar with $100 \pm 5 \mathrm{~nm}$ of regrowth with resulting $R_{\mathrm{rms}}=3 \pm 2 \mathrm{~nm}$ (not presented). Figure 2 shows the measured $\alpha$-Si thickness versus time. Previous studies ${ }^{5-7}$ measured an exponential enhancement to SPE velocity with in-plane tension. From these studies, a model was developed, proposing exponential dependence of SPE rate with in-plane stress characterized by an activation volume of $(0.15 \pm 0.01) \Omega$, where $\Omega$ is the atomic volume of Si. In order to compare the presented results with previous observations, the proposed model $^{5-7}$ was used to estimate the regrowth as a function of time. This is also shown in Fig. 2 and suggests that the regrowth observed in this study was significantly slower than expected. One possible explanation for the differences between the observed effect of tension in the present and previous studies ${ }^{5-7}$ is the presence of As, known to enhance ${ }^{14} \mathrm{SPE}$ rates. The As effect may have overwhelmed the expected enhancement from tension. However, this explanation contradicts previous results ${ }^{7}$ suggesting independent stress and dopant effects on SPE rate. In addition, it is unlikely that the presence of As is influencing volume changes between $\alpha$-Si and $\mathrm{Si}$ as previously proposed ${ }^{5,6}$ due to the similarity in size between As Downloaded 30 Mar 2009 to 131.227.178.132. Redistribution subje

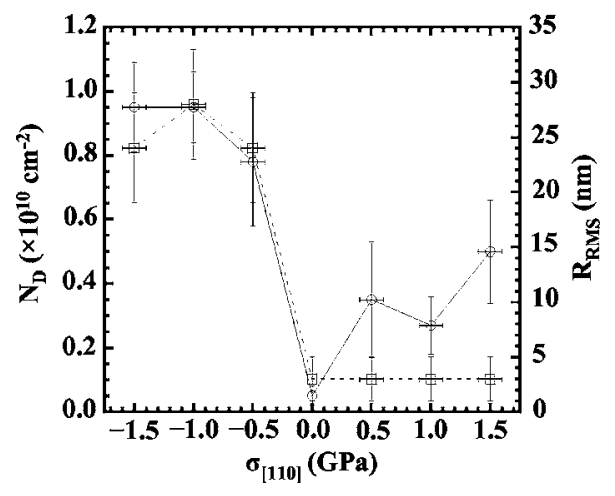

FIG. 3. Plot of $N_{D}(\bigcirc)$ after annealing for $3.2 \mathrm{~h}$ and $R_{\text {rms }}(\square)$ after annealing for $1.3 \mathrm{~h}$ vs $\sigma_{[110]}$.

and $\mathrm{Si}$ atoms and the As concentrations used. Experiments eliminating the dopant implant are in progress to further examine stress-dependent SPE rates.

Annealing for $3.2 \mathrm{~h}$ completed regrowth of the $\alpha$-Si layer in all cases as observed using WBDF-XTEM (not presented). Following SPE, the defect density $\left(N_{D}\right)$ as a function of $\sigma_{[110]}$ was estimated by assuming a sample thickness of $\sim 200 \mathrm{~nm}$ (as fixed by FIB sample preparation) and measuring the number of defects intersecting the midpoint of the regrown layer. This is plotted schematically in Fig. 3 along with the dependence of $R_{\text {rms }}$ after annealing for $1.3 \mathrm{~h}$ on $\sigma_{[110]}$. It is evident that $N_{D}$ and $R_{\text {rms }}$ in compression are significantly higher than in the stress-free or tension cases and likely correlated. However, it is interesting that $R_{\mathrm{rms}}$ is very small both in stress-free and tension cases but $N_{D}$ is significantly higher in tension relative to that in the stress-free case. Thus, it appears that roughening of the $\alpha$ /crystalline interface during SPE is not a primary mechanism responsible for SPErelated defect formation in tension. Furthermore, the observation that most of the defects nucleated near the initial $\alpha /$ crystalline interface in tension indicates that the very early stages of SPE are critically important for defect formation.

To test this theory, some specimens were partially regrown by annealing for $0.7 \mathrm{~h}$ without stress and then annealed for an additional $1.3 \mathrm{~h}$ with stress. The goal of performing the stress-free annealing was to partially regrow the layer and remove the influence of the misoriented crystallites near the original $\alpha$ /crystalline interface on subsequent stressed SPE. Figure 4(a) presents a sample annealed for $0.7 \mathrm{~h}$ without stress with subsequent annealing for $1.3 \mathrm{~h}$ with $\sigma_{[110]}=-1.5 \mathrm{GPa}$. The portion of the implanted layer regrown during the stress-free annealing is schematically indicated. The resulting $\alpha /$ crystalline interface exhibits significant interfacial roughening with nucleation of several SPErelated defects within the portion of the layer regrown with

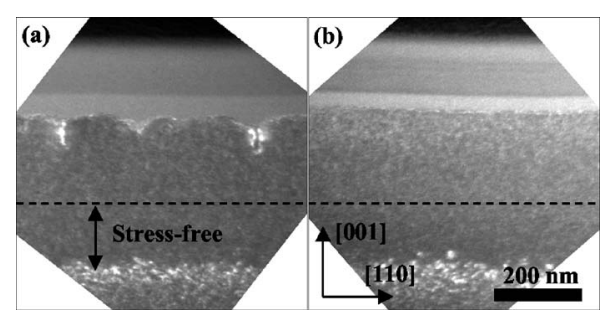

FIG. 4. WBDF-XTEM images of specimens annealed for $0.7 \mathrm{~h}$ stress-free with subsequent annealing for $1.3 \mathrm{~h}$ with $\sigma_{[110]}=(\mathrm{a})-1.5 \mathrm{and}(\mathrm{b}) 1.5 \mathrm{GPa}$.
to AIP license or copyright; see http://apl.aip.org/apl/copyright.jsp 
stress. In contrast, the specimen subjected to annealing for $1.3 \mathrm{~h}$ with $\sigma_{[110]}=1.5 \mathrm{GPa}$ after stress-free annealing for $0.7 \mathrm{~h}$, shown in Fig. 4(b), exhibits a smooth resulting $\alpha$ /crystalline interface with no SPE-related defects nucleating at the new interface.

Since misoriented crystallites ${ }^{8,12}$ near the initial $\alpha /$ crystalline interface and roughening of the regrowing interface cause SPE-related defect formation, it is plausible that stress acts to cause greater misorientation of these crystallites which accounts for the observed higher defect density in tension compared to the stress-free case. This misorientation may, in part, be driven by minimization of strain energy, since Young's modulus of $\mathrm{Si}$ is greatest along $\langle 111\rangle$ directions. ${ }^{15}$ Furthermore, very slight crystallite reorientation in $\alpha$-Si is highly plausible given the reported viscoelastic nature of $\alpha$-Si. ${ }^{16}$ Presumably, this effect occurs equally for compression and tension and thus the values of defect density in compression are indicative of both stress-induced crystallite misorientation as well as interfacial roughening. It is interesting to note the insensitivity of SPE rate with $\sigma_{[110]}$, presented in Fig. 2, though $N_{D}$ tends to increase with tension as shown in Fig. 3. Thus, since the SPE rates were very similar in all cases of $\sigma_{[110]} \geqslant 0$, the observed influence of stress on $N_{D}$ was not due to differences in SPE rates. This is important to consider since annealing in the vicinity of $\sim 450{ }^{\circ} \mathrm{C}$ is known to reduce the amount of misoriented crystallites though it is unclear if this effect is due to crystallite dissolution or SPE proceeding very slowly to allow misoriented crystallites time to reorient. ${ }^{8}$ In any case, the observations of tensile stress on defect density presented in this study indicate that the effect is not related to SPE rate.

In summary, the effect of high [110] uniaxial stresses on solid phase epitaxy and defect nucleation during regrowth of amorphous (001) Si created via ion implantation was examined. No effect on SPE velocity was observed for in-plane tension though compression did cause significant retardation. The results indicated that compressive stresses caused the greatest density of defects primarily due to roughening of the regrowing amorphous/crystalline interface. Interestingly, the application of tensile stresses caused greater defect formation compared to stress-free cases even though no significant interfacial roughening or changes in the [001] regrowth rate were observed in tension. This was possibly the result of stress-induced reorientation of crystallites near the original amorphous/crystalline interface.

The authors acknowledge the Semiconductor Research Corporation for funding this work.

${ }^{1}$ J. Vonborany and R. Kogler, Nucl. Instrum. Methods Phys. Res. A 326, 42 (1993).

${ }^{2}$ P. R. Chidambaram, C. Bowen, S. Chakravarthi, C. Machala, and R. Wise, IEEE Trans. Electron Devices 53, 944 (2006).

${ }^{3}$ C. E. Ross and K. S. Jones, Silicon Front-End Junction Formation-Physics and Technology, MRS Symposia Proceedings No. 810 (Material Research Society, Pittsburgh, 2004), p. C10.4.1.

${ }^{4}$ E. Nygren, M. J. Aziz, and D. Turnbull, Appl. Phys. Lett. 47, 232 (1985).

${ }^{5}$ W. Barvosa-Carter and M. J. Aziz, Appl. Phys. Lett. 79, 356 (2001).

${ }^{6}$ M. J. Aziz, P. C. Sabin, and G.-Q. Lu, Phys. Rev. B 44, 9812 (1991).

${ }^{7}$ W. Barvosa-Carter, M. J. Aziz, L. J. Gray, and T. Kaplan, Phys. Rev. Lett. 81, 1445 (1998).

${ }^{8}$ K. S. Jones, S. Prussin, and E. R. Webber, Appl. Phys. A: Solids Surf. 45, 1 (1988).

${ }^{9}$ L. D. Glowinski, K. N. Tu, and P. S. Ho, Appl. Phys. Lett. 28, 312 (1976).

${ }^{10}$ S. P. Nikanorov, Yu. A. Burenkov, and A. V. Stepanov, Sov. Phys. Solid State 13, 2516 (1971).

${ }^{11}$ C. R. Olson, E. Kuryliw, B. E. Jones, and K. S. Jones, J. Vac. Sci. Technol. B 24, 446 (2006).

${ }^{12}$ T. Sands, J. Washburn, E. Myers, and D. K. Sadana, Nucl. Instrum. Methods Phys. Res. B 7-8, 337 (1985).

${ }^{13}$ L. Csepregi, E. F. Kennedy, and J. W. Mayer, J. Appl. Phys. 49, 3906 (1978).

${ }^{14}$ J. S. Williams and R. G. Elliman, Phys. Rev. Lett. 51, 1069 (1983).

${ }^{15}$ J. J. Wortman and R. A. Evans, J. Appl. Phys. 36, 153 (1976).

${ }^{16}$ C. A. Volkert, J. Appl. Phys. 70, 3521 (1991). 